
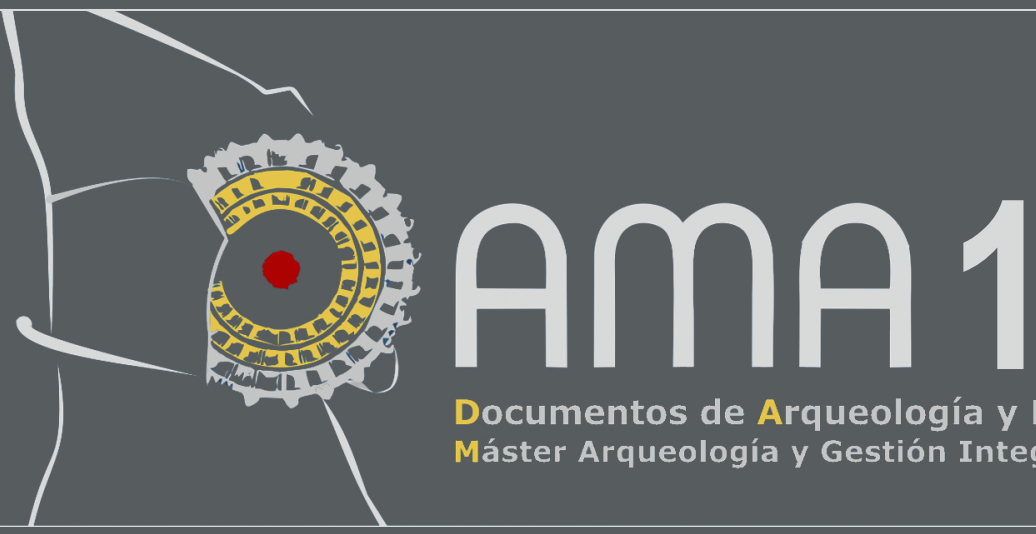

Documentos de Arqueología y Patrimonio Histórico

Máster Arqueología y Gestión Integral del Patrimonio de la Universidad de Alicante

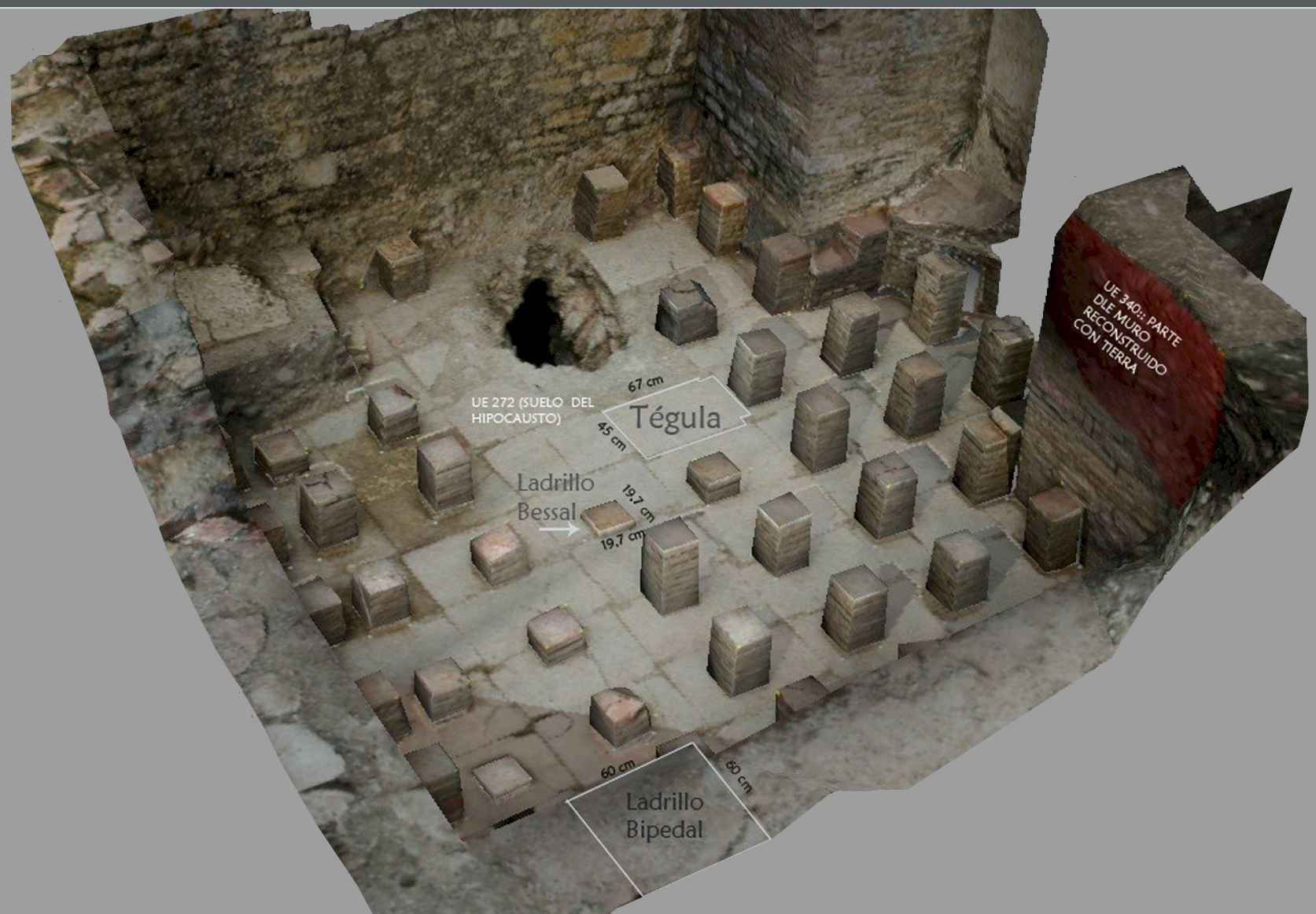

Documentos de Arqueología y Patrimonio Histórico Revista del Máster Universitario en Arqueología Profesional y Gestión integral del Patrimonio 



\section{DAMA 1}

\section{6}

DOCUMENTOS DE ARQUEOLOGÍA Y PATRIMONIO HISTÓRICO DEL MÁSTER UNIVERSITARIO EN ARQUEOLOGÍA PROFESIONAL Y GESTIÓN INTEGRAL DEL PATRIMONIO DE LA UNIVERSIDAD DE ALICANTE 


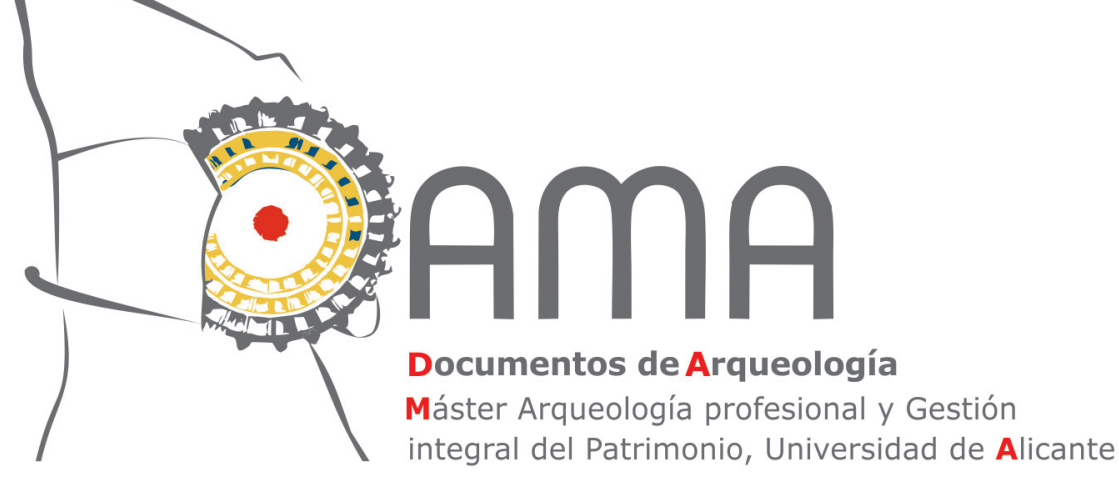

La revista electrónica DAMA. Documentos de Arqueología y Patrimonio Histórico surge como servicio para todos aquellos alumnos del Máster en Arqueología Profesional y Gestión Integral del Patrimonio de la Universidad de Alicante que se están iniciando en la investigación y cuya primera aportación a nuestra disciplina suele ser su Trabajo de Fin de Máster (TFM). Estos proyectos en muchos casos representan casi todo un curso de trabajo y esfuerzo, y con frecuencia quedan inéditos.

El objetivo de esta revista es ofrecer un medio que facilite la publicación de los resultados de sus TFM. La edición se presenta en versión digital y cuenta con su correspondiente ISSN. Se publica de forma anual en el sitio web de la Universidad de Alicante (http://web.ua.es/es/dama/) y en su repositorio (RUA). Los artículos publicados son descargables en formato PDF.

\section{Consejo de Redacción}

Directores (Coordinadores del Máster)

Carolina Doménech Belda

Fernando Prados Martínez

\section{Secretaria de Edición}

Julia Sarabia Bautista

\section{Vocales}

Los miembros de la Comisión Académica del Máster Universitario en Arqueología Profesional y Gestión del Patrimonio (http://dprha.ua.es/es/magip/comision-academica.html)

\section{Edita}

Máster Universitario en Arqueología Profesional y Gestión Integral del Patrimonio Departamento de Prehistoria, Arqueología, Historia Antigua, Filología Griega y Filología Latina

Facultad de Filosofía y Letras II

Universidad de Alicante

Ctra San Vicente del Raspeig s/n

E-03690 San Vicente del Raspeig (Alicante)

Web:http://dprha.ua.es

Teléfono: (+34) 965903663

Fax: (+34) 965903823

E-mail: revista.dama@ua.es

\section{ISSN}

$2530-2345$

\section{Portada}

Modelo fotogramétrico de un hypocaustum romano realizado por Ana Charquero 
EDITORIAL

Coordinación del máster

ENTREVISTA A SOLVEIG NORDSTRÖM: UNA MIRADA HACIA EL PASADO DE LA ESCUERA

Raúl Berenguer González ..

\section{ARQUEOLOGÍA Y MÉTODO}

EL ESTUDIO DE LOS MATERIALES CONSTRUCTIVOS DE TIERRA DEL CABEZO DEL POLOVAR (VILLENA, ALICANTE): APORTACIÓN A LAS FORMAS CONSTRUCTIVAS DE DOS PEQUEÑAS EDIFICACIONES CAMPESINAS DE LA EDAD DEL BRONCE EN EL LEVANTE PENINSULAR

María Pastor Quiles

VIVIENDAS POSTALAYÓTICAS: UNA APROXIMACIÓN A LOS ESPACIOS DOMÉSTICOS EN EL ARCHIPIÉLAGO BALEAR (550-123 ANE)

Octavio Torres Gomáriz

LAS FLOTAS DE GUARNICIÓN ROMANA EN LAS COSTAS DE HISPANIA A PARTIR DE LAS FUENTES EPIGRÁFICAS

Sergio Lledó Ramírez

UNA APROXIMACIÓN A LA RECONSTRUCCIÓN DEL PAISAJE HISTÓRICO: EL PROYECTO L'ALMISSERÀ Diana López Arroyo

NUEVAS PERSPECTIVAS PARA EL ESTUDIO ARQUEOLÓGICO DEL POBLAMIENTO RURAL MEDIEVAL EN ASPE (ALICANTE): HUERTAS Y ALQUERÍAS JUNTO AL RÍO TARAFA

Felipe Mejías López

ARQUEOLOGÍA DE LAS GUERRAS CARLISTAS

Iván Roldán Vergarachea

LA CERÁMICA DE USO ARQUITECTÓNICO EN NOVELDA: LA AZULEJERÍA DE FINALES DEL SIGLO XIX Y PRINCIPIOS DEL XX

Natalia Sala Pérez

PRÁCTICA Y USOS DE LA FOTOGRAMETRÍA DIGITAL EN ARQUEOLOGÍA

Ana $M^{a}$ Charquero Ballester.

\section{GESTIÓN Y PUESTA EN VALOR DEL PATRIMONIO}

ARQUEÓLOGOS CON DISCAPACIDAD. ARQUEOLOGÍA INCLUSIVA

Ana Samaniego Espinosa

"LES COVES DELS PESCADORS DE EL CAMPELLO": PROPUESTA DE RECUPERACIÓN, PUESTA EN VALOR Y USO PÚBLICO DE UN PATRIMONIO OLVIDADO

Ana Isabel Castro Carbonell .

LA COLONIA DE SANTA EULALIA. ESTUDIO Y PROPUESTA DE RECUPERACIÓN

Héctor de Arriba González

PROYECTO DE PUESTA EN VALOR DE LOS RESTOS DE LA GUERRA CIVIL EN LA CIUDAD DE ALICANTE

Leticia Victoria González Chouciño

ANTEPROYECTO MUSEOGRÁFICO DEL MUSEO DE HISTORIA DE SAX

Alberto Ochoa García

EL CONGRESO DE MÁSTER: UNA PROPUESTA DIDÁCTICA DEL MÁSTER DE ARQUEOLOGÍA DE LA UNIVERSIDAD DE ALICANTE

Ignasi Grau Mira, Sonia Gutiérrez Lloret, Carolina Doménech Belda, Julia Sarabia Bautista 



\title{
PROYECTO DE PUESTA EN VALOR DE LOS RESTOS DE LA GUERRA CIVIL EN LA CIUDAD DE ALICANTE
}

\author{
Leticia Victoria González Chouciño
}

\section{RESUMEN}

En este artículo se presenta una propuesta de puesta en valor de los restos de la Guerra Civil que se encuentran en la ciudad de Alicante, así como de los lugares de la memoria. Algunos de estos restos y lugares son: los refugios que se encuentran por la ciudad, la defensa antiaérea y trincheras de la Serra Grossa y lugares de la memoria como el Mercado Central, el Campo de los Almendros y el Puerto. En España, la puesta en valor y estudio de este patrimonio es algo reciente, a diferencia de otros países, donde el patrimonio bélico hace tiempo que se estudia y pone en valor. En el caso de Alicante este año se han musealizado dos refugios y se ha creado un centro de interpretación. Este proyecto pretende integrar esos refugios y el centro de interpretación en un itinerario que aglutine, además, los principales restos y lugares vinculados a la guerra civil que se encuentran en la ciudad de Alicante. La finalidad es crear un discurso que enseñe lo sucedido en dicha contienda a la sociedad y que además sea una forma de crear un turismo cultual que sirva como alternativa al turismo de sol y playa.

Palabras clave: Guerra Civil, Alicante, puesta en valor, patrimonio, divulgación.

\section{ABSTRACT}

In this article we present an idea for the enhancement of cultural heritage of the rests of the Spanish Civil War in Alicante city, and also memory space. Some of this rests and places are: air raid shelters around the city, the anti-aircraft warfare and the trenchs in Serra Grossa and memory space as Mercado Central, Campo de los Almendros or the Harbour. Unlike in other countries, enhancement of cultural heritage and the research of this heritage is a new phenomenon in Spain. In the case of Alicante, this year two air raid shelters have become museums and an interpretation centre has been created. The aim of this project is to integrate these shelters and the interpretation centre in one itinerary which also includes the main rests and places related to the Spanish Civil War of Alicante city. The purpose is to creat a discourse which shows to society what happened in this war, and also to create a tourism based in culture and not just in sun and beach.

Key words: Spanish Civil War, Alicante, enhancement of cultural heritage, heritage, dissemination. 


\section{INTRODUCCIÓN: PROYECTO DE PUESTA EN VALOR}

En nuestro país hay varios ejemplos de la puesta en valor del patrimonio material de la Guerra Civil. Lo más habitual en contextos urbanos es la musealización de refugios antiaéreos, por su gran número, su buen estado de conservación, así como por su atractivo. Podemos encontrar dos tipos de puesta en valor de estos refugios, los que se muestran tan cual intentando preservar su estado original y los que utilizan recursos museográficos para contar lo acontecido en la guerra (Besolí, Peinado, 2008, p. 11).

Uno de estos ejemplos lo podemos encontrar en la ciudad de Barcelona donde hay varios refugios visitables. Esta ciudad llegó a contar con unos 1400 refugios para proteger a la población. Uno de ellos es el refugio 307 del Poble Sec, situado en el pie de la montaña de Montjuic, con una capacidad para 2.000 personas, fue abierto al público en el año 2007. De su gestión se encarga el Museu d'Història de Barcelona. Otro de los refugios visitables de Barcelona es el del Palacio de les Heures, con un excelente estado de conservación, pues incluso se conserva la instalación eléctrica. También es importante el refugio que se encuentra debajo de la Plaza del Diamante, que fue reabierto en el 2006, contaba con una capacidad para 200 personas que se distribuían en 200 metros de estrechos túneles. Su estado de conservación es muy bueno, pudiéndose apreciar aun los bancos de piedra adosados a las paredes, los sanitarios y las marcas de las velas que la gente utilizaba para iluminar. Otro ejemplo en esta ciudad es el del refugio que se encuentra en el barrio de Gracia en la Plaza de la Revolución, actualmente solo se puede visitar una pequeña parte formada por una galería y dos salas de enfermería (Ibidem, p. 10). Todos estos refugios se pueden visitar pero debe ser con guías, y no forman parte de una misma ruta, pues cada refugio es gestionado por distintas entidades, tanto públicas como privadas.

En la provincia de Almería se musealizó un refugio antiaéreo que también se utiliza como centro de exposiciones. Tiene el atractivo de poseer espacios recreados: almacén despensa, un quirófano, con todo el instrumental de la época. Fue rehabilitado por el Ayuntamiento de Almería, es uno de los refugios abiertos al público más grande de Europa.

Otro de los ejemplos que encontramos en nuestro país es el de la ciudad de Cartagena, donde en el año 2004 se rehabilitó, dentro del proyecto "Cartagena, Puerto de Culturas", el refugio antiaéreo construido en el Cerro de la Concepción. Se trata de galerías subterráneas excavadas directamente en la roca del cerro, su capacidad era para aproximadamente unas 5500 personas (Ibidem, pp. 11-12).

En nuestra provincia también hay ejemplos de puesta en valor de este patrimonio, concretamente en la ciudad de Alcoy donde se musealizó en el año 2006 el Refugio de Cervantes, mide más de 100 metros de longitud donde se han puesto paneles y fotografías que explican la Guerra Civil en Alcoy, también hay videos y objetos militares, así como otros recursos como sonido de explosiones. Este año en la ciudad de Alicante se han abierto al público dos de los 94 refugios antiaéreos conocidos, el de la plaza Balmis y unos meses después el de la plaza Séneca junto al Centro de Interpretación de los Refugios Antiaéreos.

En muchas otras poblaciones de nuestro país existen refugios que se encuentran en un buen estado de conservación, solo se abren a visitas en momentos concretos. Con estas iniciativas se busca que el visitante sienta empatía y tome consciencia de las condiciones en las que fueron utilizados durante la Guerra Civil (Ibidem, p. 10). 
Además de los refugios también podemos encontrar algunas rutas que hacen un recorrido por distintos elementos patrimoniales de la Guerra Civil. Así tenemos en Aragón la llamada "Ruta Orwell" en el frente de Monegros donde se pueden ver el frente de guerra, hay elementos como búnkeres, trincheras, lugares de descanso de los soldados, alambradas, etc. También en Aragón en la Comarca del Jiloca, podemos encontrar otra ruta donde hay numerosas trincheras, nidos de ametralladora, refugios, búnkeres. Otra ruta es la del parque Lineal de Madrid donde, al igual que la ruta anteriormente mencionada, se pueden ver trincheras. También en la Comunidad de Madrid, en el Valle Bajo del Lozoya, Sierra de Guardarrama podemos encontrar otra ruta histórica, en ella se pueden visitar observatorios blindados, fortines tipo blockhaus y cruciformes, paramentos, nidos de ametralladoras, miradores fortificados y trincheras. Estas rutas suelen darte en entornos naturales combinándose con rutas medioamentales, cuentan con paneles explicativos y folletos. En entornos urbanos se pueden encontrar recorridos sobre la Guerra Civil hechos por iniciativa privada como es el caso de la ciudad de Barcelona o Madrid.

En este proyecto se busca la singularidad, porque si bien en numerosas ciudades en nuestro país se pueden visitar refugios antiaéreos, no en todos se ofrece la visita a otros lugares relacionados con la Guerra Civil, como puede ser la defensa activa, así como lugares de represión posteriores a la guerra y la visita a sitios históricos importantes, todo en una misma ruta, con un mismo discurso y en una extensión relativamente corta, combinándose con la red de senderos urbanos de la ciudad. Esto da a este proyecto una particularidad que no se ve en otras ciudades de España y que puede hacerlo muy atractivo teniendo también en cuenta el creciente interés en este tipo de patrimonio en los últimos años.

\section{PROPUESTAS GENERALES 2.1. Paneles}

Los paneles serán el recurso interpretativo que se utilizará en cada uno de los lugares que se pondrán en valor. Es el recurso más importante a la hora de difundir la historia de cada uno de los sitios. Constarán de los siguientes elementos:

a) Un número identificativo que tendrá una relación con cada uno de los lugares de la ruta.

b) Un mapa donde se marquen todos los lugares de la ruta con su correspondiente número indicando la ubicación.

c) Algunas líneas explicando lo allí acontecido y su importancia en el contexto de la Guerra Civil en Alicante.

d) Información adicional que requiera cada uno de los lugares, como puede ser horario de visita específico.

e) Imágenes que estén en relación con ese bien, como pueden ser fotografías, plantas, secciones, representaciones en 3D, etc.

f) Dos códigos QR. Uno de los códigos permitirá descargar un archivo de audio donde se relata una historia contada en primera persona relacionada con ese lugar. En cada uno de los paneles se descarga una parte diferente de la historia, por lo que es necesario visitar todos los lugares para conocerla entera. El otro código QR permitiría acceder a una página web con toda la información referente a cada uno de los lugares, así como a los distintos mapas de la ruta. 
También es importante tener en cuenta las características físicas que debe tener el panel:

a) Para evitar el deterioro de los paneles a causa del vandalismo, especialmente en zonas no vigiladas. Algo que ya ha ocurrido con elementos relacionados con la Guerra Civil, como por ejemplo en las placas conmemorativas colocadas por la Comisión Cívica por la recuperación de la memoria histórica en el Mercado, Puerto y en el Campo de los Almendros. Se utilizaría un material como es el HPL, que se ha estado probando en La Villajoyosa con bastante éxito. Este material resiste muy bien las inclemencias del tiempo y, en caso de pintadas vandálicas, se limpia con mucha facilidad.

b) El color de fondo del panel debe ser oscuro pues estarán al aire libre y en caso de que les dé el sol los colores oscuros de fondo facilitan la lectura con respecto a un fondo claro.

c) El panel debería tener de altura de 0,70 metros.

\subsection{Folletos}

Otro de los recursos más importantes para difundir este tipo de patrimonio es el uso de los folletos, estos se distribuirían en oficinas de información turística y en el centro de interpretación de los "Refugios Antiaéreos". En los folletos vendría toda la información necesaria, como puede ser el horario de visita de algunos de los lugares, el horario de las visitas guiadas así como el mapa con los distintos recursos a visitar. Así como un código QR para acceder a la página web con toda la información referente a la ruta y a los distintos lugares.

Este recurso puede ser muy útil que, como ya se ha mencionado antes, esté disponible en el Centro de Interpretación, de esta manera el visitante del centro podrá introducirse en el contexto de la Guerra Civil tanto a nivel general como a nivel provincial y a nivel local. Para posteriormente, teniendo como recurso el folleto, poder visitar los distintos lugares que se encuentran en el itinerario, ya sea siguiendo un orden cronológico o por cercanía.

\subsection{Recursos en Internet}

Hoy en día es importante para la difusión de cualquier producto el tener presencia en la red. Sería necesario disponer de una página web desde donde se pueda acceder con los códigos QR que aparecerán en paneles y folletos, para poder desde ahí acceder a toda la información referente a los distintos lugares así como descargar las rutas y los archivos de audio. También se podría tener presencia en las distintas redes sociales para dar una mayor difusión y poder subir noticias relacionadas con este patrimonio.

\subsection{Personal}

En algunos de los lugares es necesaria la presencia de un guía para poder ser visitados, como es el caso de los refugios y los depósitos de la CAMPSA. El guía también es necesario para las visitas guiadas a los distintos puntos que se encuentran en los itinerarios, yendo con un mismo guía de un punto a otro para explicar lo acontecido durante la Guerra Civil en cada uno de los lugares de la ruta.

\subsection{Entrada única}

Para mayor comodidad de la visita se vendería una entrada única que permitiría acceder al Centro de Interpretación, a los tres refugios antiaéreos, a los depósitos de la CAMPSA, así como viajes gratuitos en las líneas del TRAM y el acceso al ascensor del Castillo de Santa Bárbara. Además de la posibilidad de realizar los dos itinerarios completos con un guía. 


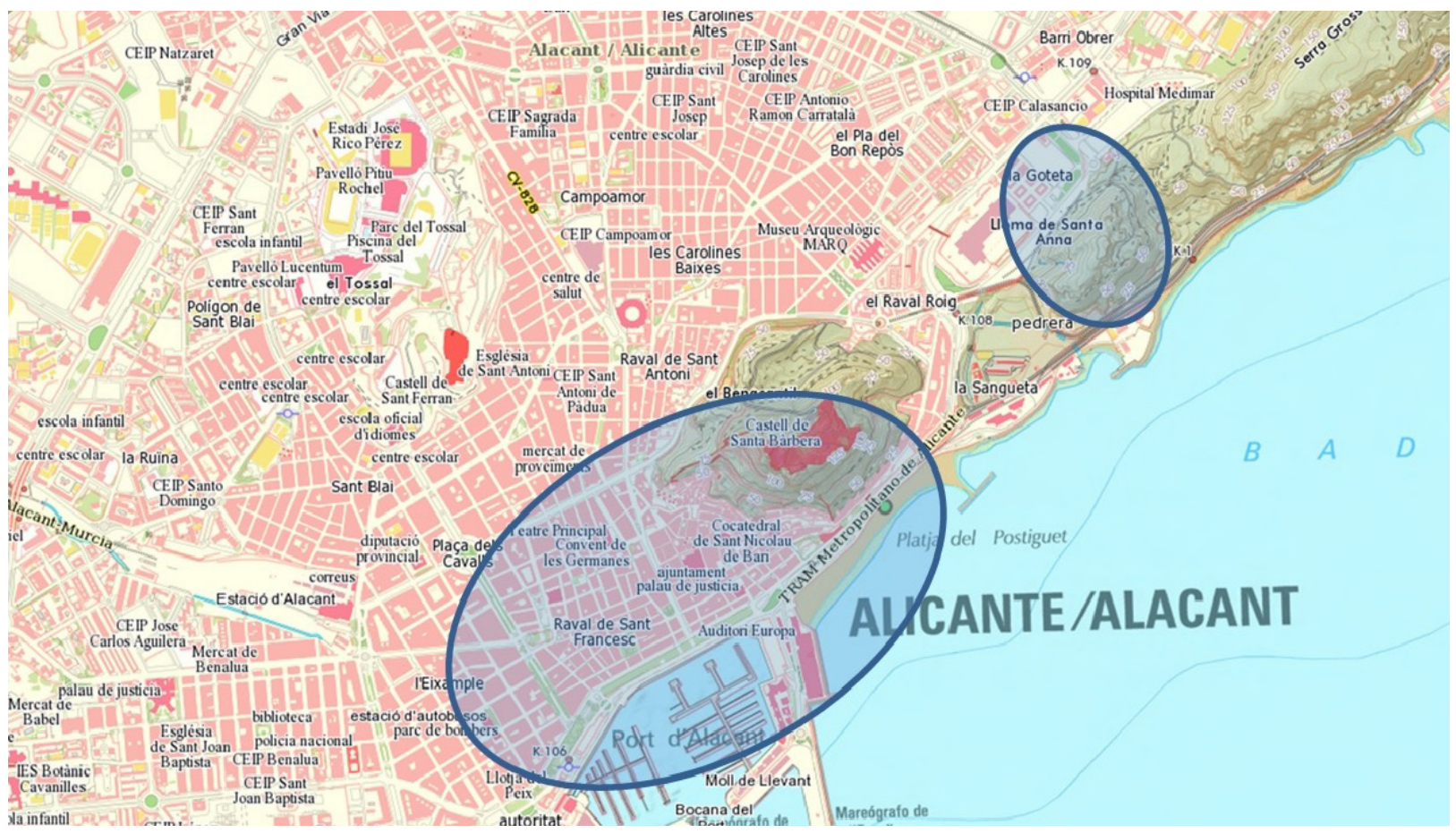

Fig. 1. Mapa de la ciudad de Alicante donde se señalan las zonas donde se encuentran los distintos lugares que forman parte de los itinerarios. Fuente: http://terrasit.gva.es/es/ver

\subsection{Itinerarios}

Se crearán dos itinerarios que unan los distintos lugares (Fig. 1) para que el visitante que desee visitarlos en un solo día o dos pueda hacerlo en un orden cómodo, o para que al menos sepa cómo llegar de un sitio a otro. Para esto se podrá combinar distintos tipos de medio de transporte. Cada uno de los itinerarios dura aproximadamente unas 2 horas, en el caso de que se hagan con guía, y el tiempo que se tarda en llegar de un punto al siguiente del itinerario es de unos 8 minutos de media.

\subsubsection{Itinerario 1}

Este itinerario (Fig. 2) irá por el centro de la ciudad de Alicante y partirá desde el Centro de Interpretación,

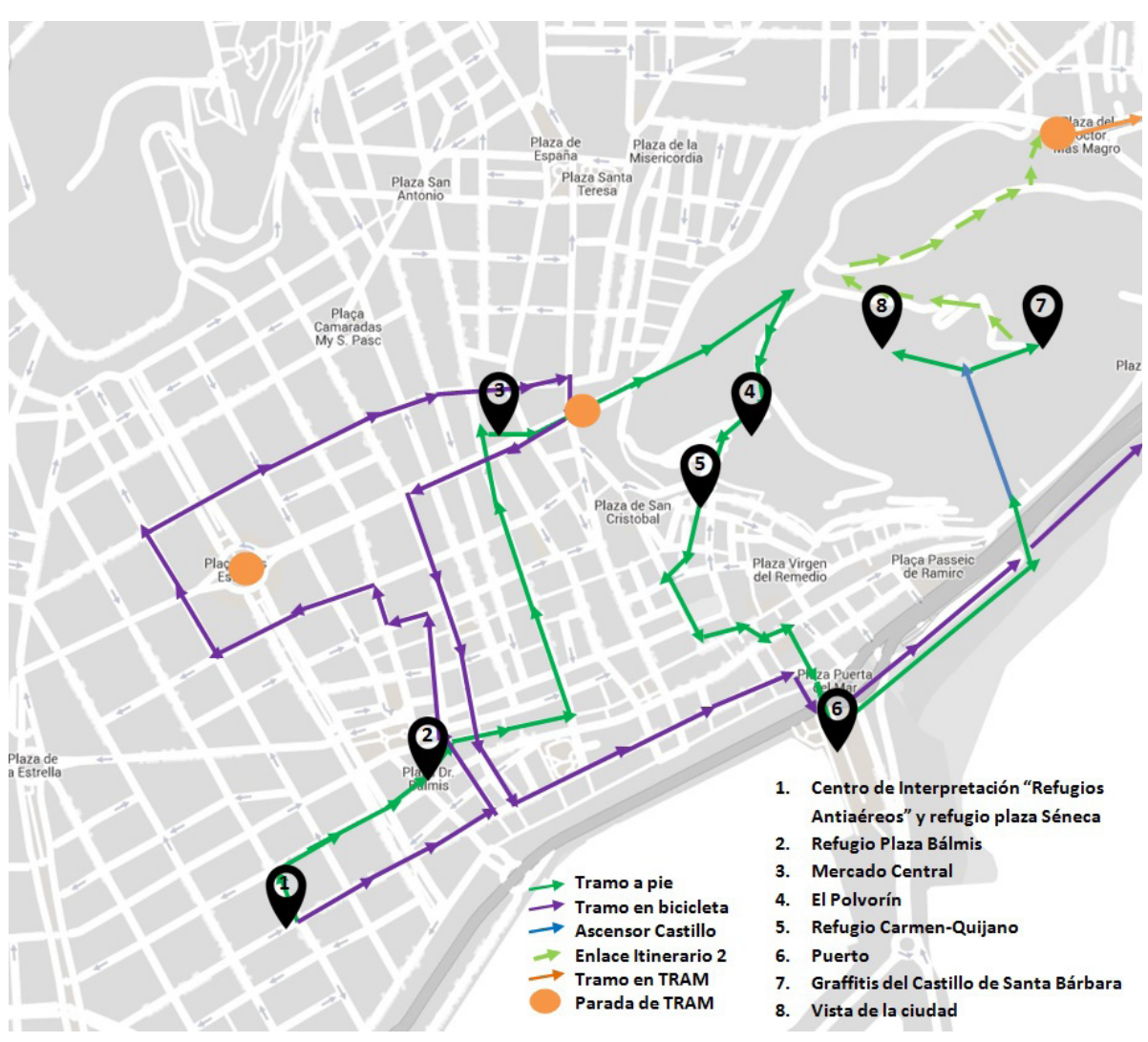

Fig. 2. Propuesta de itinerario 1. donde el visitante podrá informarse sobre la Guerra Civil en general para ponerse en contexto y sobre la guerra en Alicante. 
En esta ruta se podrán ver tres refugios, el Mercado Central, la zona de la defensa antiaérea, el Puerto y el Castillo de Santa Bárbara, por lo que solo haciendo esta ruta se pueden aprender acerca de la defensa pasiva, la activa, los bombardeos y la represión.

\subsubsection{Itinerario 2}

El segundo itinerario (Fig. 3) propuesto comienza en la parada de TRAM de La Sangueta, para dirigirse en primer lugar a los depósitos subterráneos de la CAMPSA. Desde allí se continuará subiendo a la Serra Grossa con la finalidad de ver parte de la defensa antiaérea. Hay que señalar que esto conlleva algo de dificultad al itinerario que podría impedir la accesibilidad de la totalidad de los visitantes. Finalmente el itinerario concluye con la visita al campo de concentración de los Almendros, que al estar junto a la parada de TRAM de la Goteta-Plaza Mar 2, facilita la comunicación hacia el centro de la ciudad y permite la visita a aquellos que no quieran o puedan subir a la Serra Grossa.

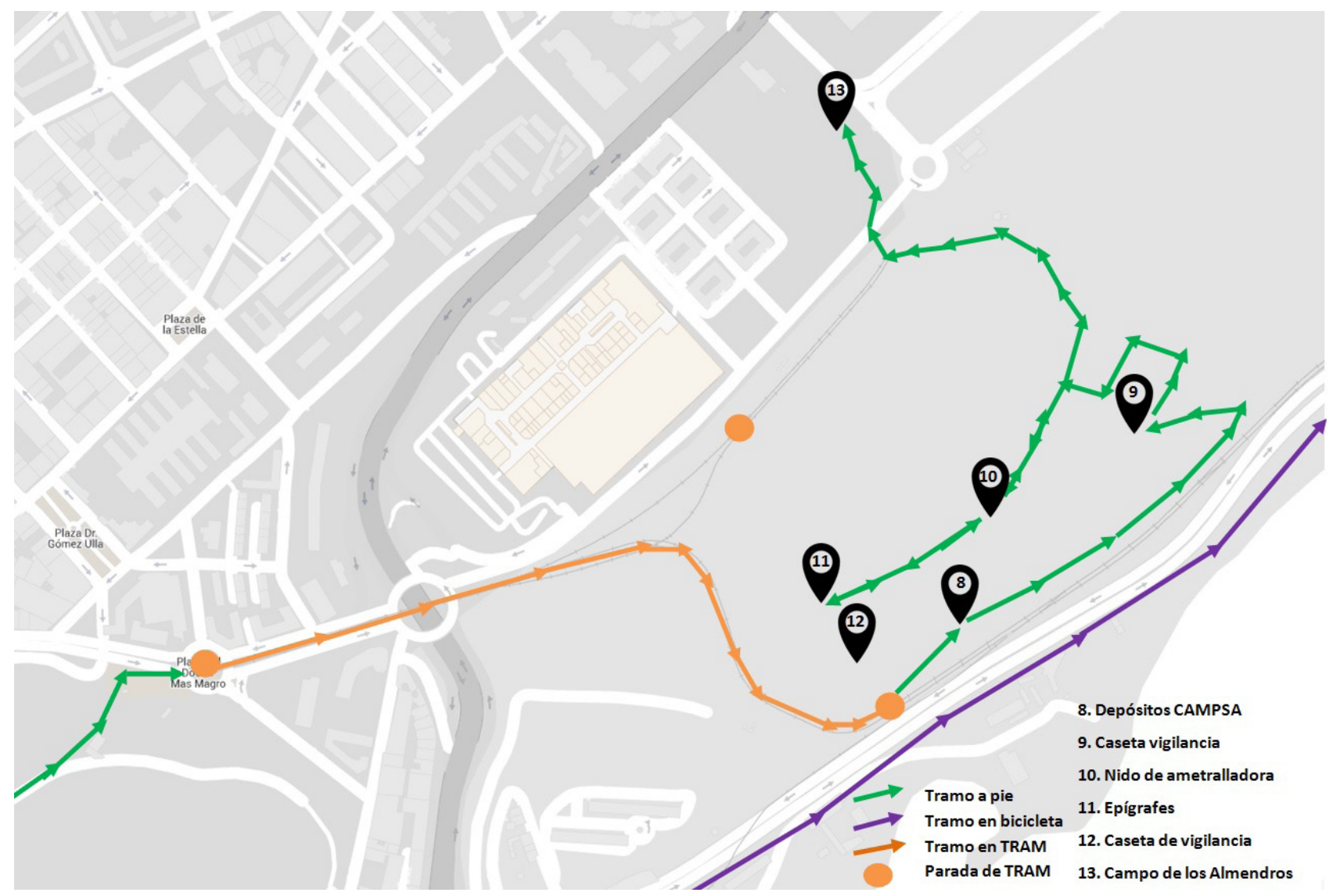

Fig. 3. Propuesta de itinerario 2.

Ambos itinerarios se pueden realizar en un mismo día, pues se puede combinar el Itinerario 1 con el 2 bajando por el castillo, visitando lo que sería uno de los senderos urbanos de Alicante, y cogiendo el TRAM en la parada de MARQ-Castillo hacia la parada de La Sangueta por las líneas L1, L2 y L4. También se puede realizar cada itinerario en días diferentes, o solo realizar uno de ellos, pues en ambos itinerarios se ven temas similares, como son los refugios, la defensa antiaérea, los bombardeos y la represión. 


\subsection{Señalización por la ciudad}

En distintos puntos de la ciudad se podrían instalar señalizaciones que nos indiquen la cercanía a alguno de los puntos del itinerario y su dirección.

\subsection{Ruta a pie, senderismo}

Buena parte del itinerario se puede realizar a pie, como es el caso de los lugares que se encuentran en la zona centro de Alicante, pudiendo combinar esta visita con el transporte público. También está la posibilidad de combinar esta ruta a pie con los Senderos Urbanos existentes en Alicante, que fueron creados por Medio Ambiente para conocer el patrimonio natural de la zona; dentro de estos se debe incluir el patrimonio de la Guerra Civil y así combinar ambos itinerarios. Estos itinerarios ya existentes son, primero, el de la Serra Grossa, donde se encuentra la defensa antiaérea, los depósitos de la CAMPSA, así como el campo de los Almendros. El del parque de la Ereta con el Polvorín y el del Monte Benacantil con el Castillo y sus graffitis.

La ruta a pie por el caso urbano pasaría por las calles peatonales más populares de la ciudad, como la calle San Francisco y la calle Castaños. También pasaría por lugares importantes de la ciudad como el Barrio Santa Cruz, la catedral de San Nicolás, el Ayuntamiento de Alicante y la playa del Postiguet.

\subsection{Ruta en bicicleta}

Alicante cuenta desde hace unos años con carril bici que permite ir a casi cualquier parte de la ciudad en bicicleta; estos carriles también pueden aprovecharse para crear una ruta en bicicleta para quien prefiera visitar los distintos lugares con este medio de transporte. Esta ruta sería necesaria combinarla en parte con la ruta en TRAM y con partes a pie.

\subsection{Ruta en TRAM}

El TRAM de Alicante cuenta con varias paradas que se encuentran muy cercanas a los lugares del itinerario, estas son la parada del Mercado, la del MARQ-Castillo, la de la Goteta-Plaza Mar 2 (junto a la Serra Grossa y el campo de los Almendros) y Sangueta (junto a los antiguos depósitos de la CAMPSA).

Para el uso del TRAM se podrían emitir billetes especiales combinados con la entrada al Centro de Interpretación, así como la visita a otros lugares de esta ruta, como ya se hace en Alicante con el MARQ y dos de sus yacimientos; La Illeta dels Banyets de El Campello y Lucentum en la Albufereta.

\subsection{Accesibilidad}

Estos itinerarios cuentan con algunas dificultades de acceso. En el primer itinerario propuesto la dificultad es escasa a excepción de la visita al Polvorín, en el parque de la Ereta. En el segundo itinerario la parte de la Serra Grossa es relativamente poco accesible. Para solucionar este problema se pueden hacer rutas alternativas pero también intentar mejorar los accesos a las distintas partes de la ruta.

\section{PROPUESTAS CONCRETAS}

Para el estudio de cada uno de los lugares que se pretenden incluir en los itinerarios se llevó a cabo una serie de fichas (ejemplo: figura 4) donde se recogían los datos más relevantes. Entre estos datos podemos encontrar la ubicación, una descripción detallada y su historia, su titularidad, su 
nivel de protección (en caso de no tenerlo se propone uno), así también una propuesta de actuación, bibliografía e imágenes tanto antiguas como actuales. De esta forma poder presentar en el trabajo los datos más importantes de cada uno de los sitios utilizados para esta ruta cultural.

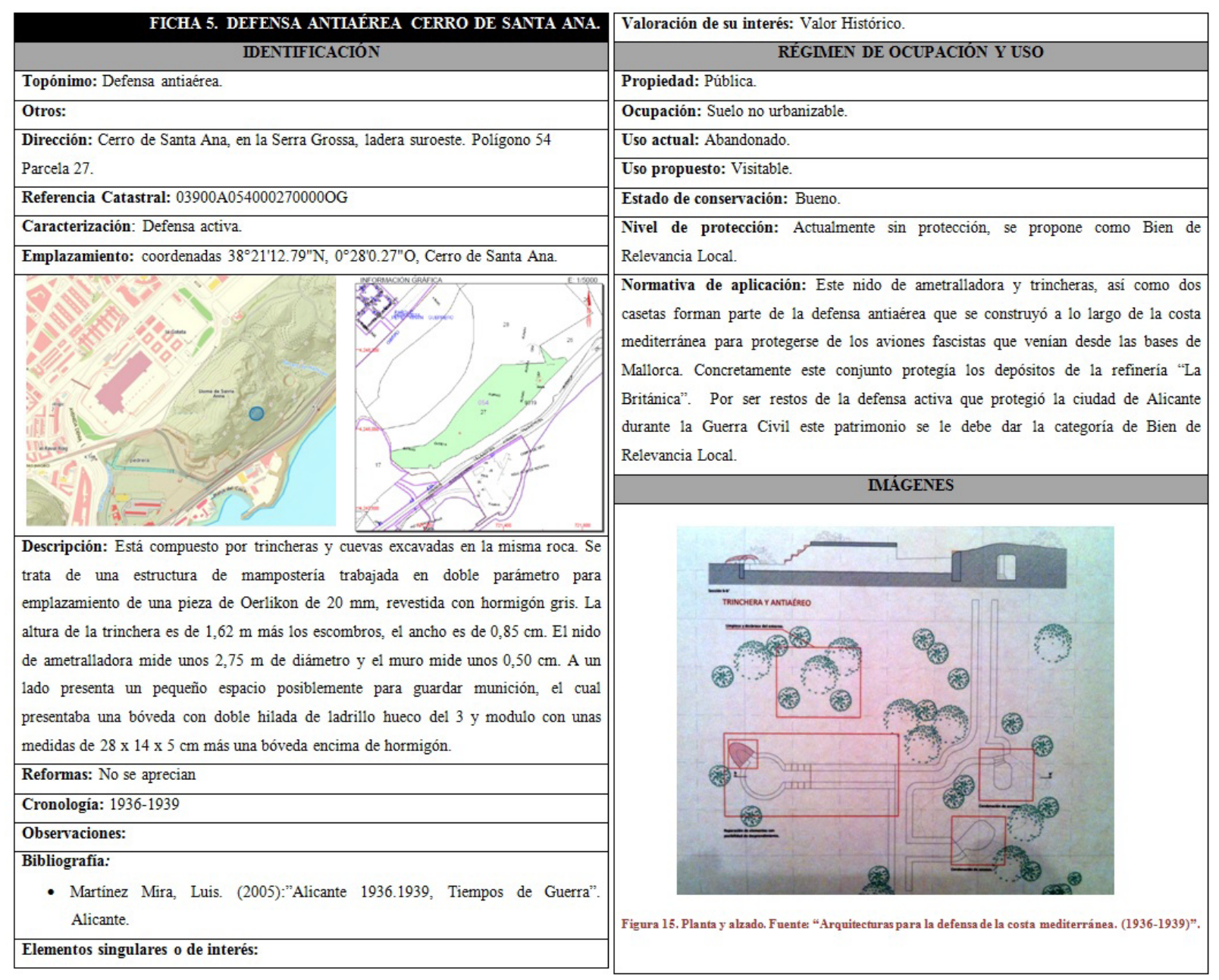

Fig. 4. Ejemplo de ficha de catalogación.

\subsection{Refugio}

Se propone la musealización de al menos un refugio mas, aparte de los ya musealizados en la plaza Bálmis y en la plaza Séneca. Serian adecuados para su musealización cualquiera de los dos ubicados en el casco antiguo de la ciudad, el de la plaza Quijano-Carmen (Fig. 5) y el aparecido en la calle General Rovira ya que se encuentran en un buen estado de conservación. Para la puesta en valor de alguno de ellos se propone, además del panel instalado fuera como en todos los demás puntos del itinerario, la instalación de algún sistema que permita la proyección de sombras de personas en las paredes para crear la sensación al visitante de que el refugio se encuentra lleno de gente. También se propone a modo de homenaje proyectar al final de la visita los nombres que se conozcan de las víctimas de los bombardeos. Para poder visitar este refugio será necesaria la entrada con un guía.

\subsection{CAMPSA}

Su puesta en valor consistiría principalmente en habilitar este lugar para su visita al público, pues estos antiguos depósitos se encuentran en un estado de conservación bueno, pero requeriría una limpieza y consolidación de algunos elementos para que la visita pueda ser segura, así como la instalación de iluminación. 


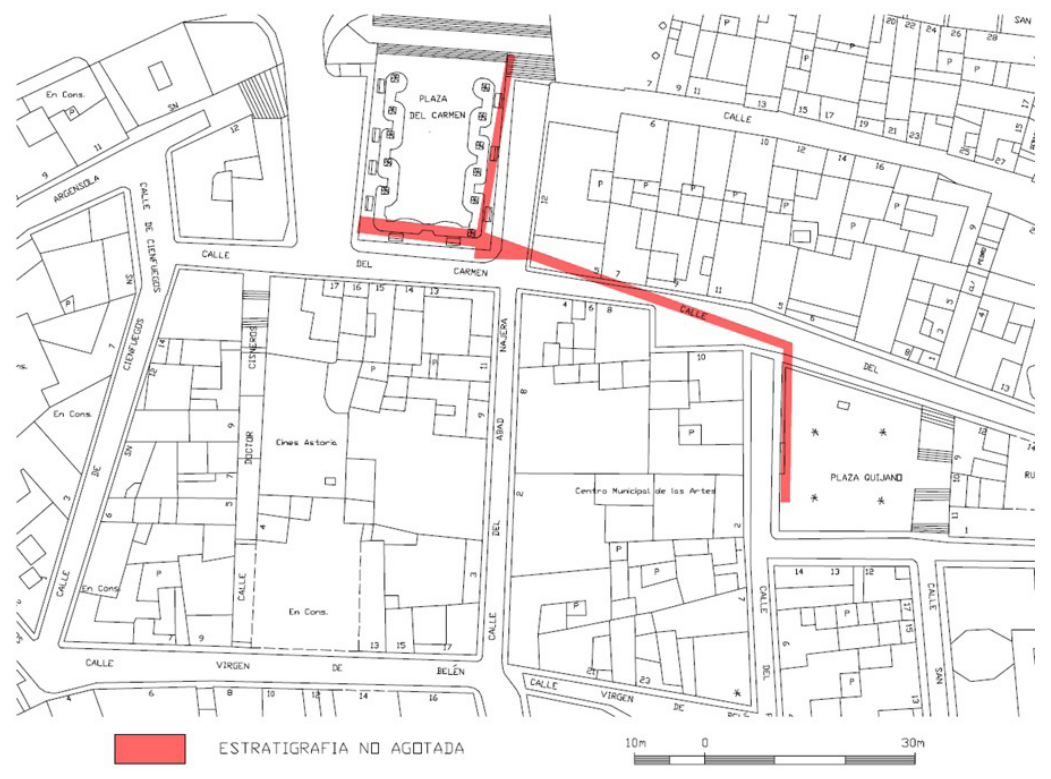

Fig. 5. Situación del refugio. Fuente: P.E.P.A.

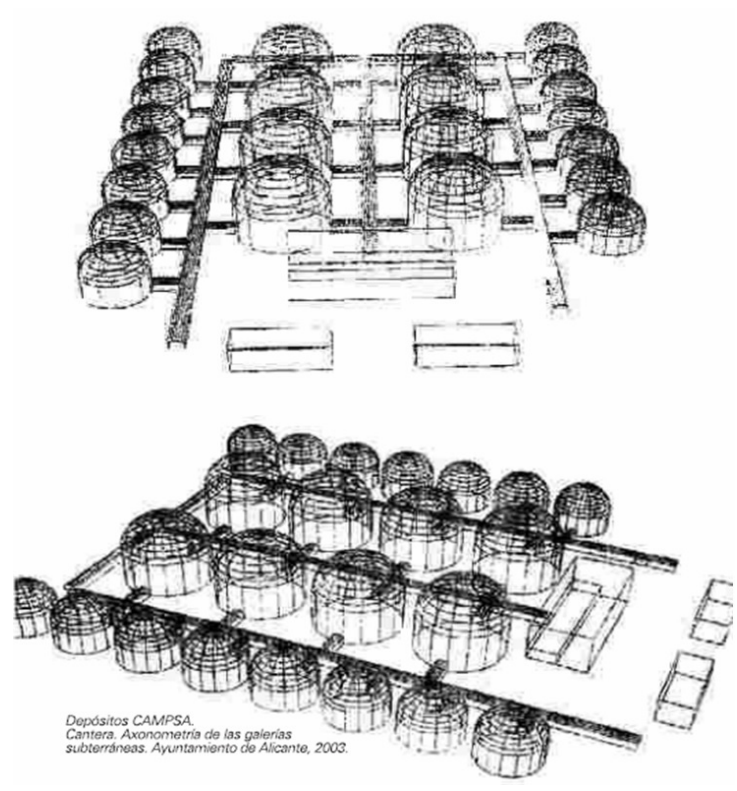

Fig. 6. Axonometría de las galerías (Aguilar, 2006, p. 30).

Este lugar por sí solo sin nada añadido ya es lo suficientemente atrayente como para poder ser visitado (Fig. 6). Las visitas deberán realizarse en grupos reducidos con un guía que vaya explicando la historia de la Refinería "La Británica" y su importancia en la Guerra Civil. A los visitantes se les debe dotar con cascos de seguridad y linternas.

En la entrada de los depósitos se pondría un panel explicativo al igual que en los demás sitios, en este caso también con toda la información para poder realizar una visita.

\subsection{Serra Grossa}

En la Serra Grossa la situación es más compleja que en los demás sitios, pues nos encontramos con una zona menos accesible y con unos restos que requieren de consolidación y restauración.

Aunque es accesible, la Serra Grossa consta de dificultades debido a su pendiente y terreno no asfaltado que podría impedir el acceso a determinados visitantes, personas con discapacidades podrían tener muchos problemas en acceder a esta zona. Es necesario acondicionar los caminos que no se encuentran en buen estado, así como colocar vallas, que agredan lo menos posible el paisaje, en determinadas zonas donde los caminos son más estrechos.

Dentro de esta zona hay tres elementos de interés:

3.3.1. Nido de ametralladora con trincheras (Fig. 7): Se encuentra en buen estado pero es necesaria una limpieza, consolidación y restauración. Deberían instalarse unas vallas en las zonas con más pendiente. Se pondrá el correspondiente panel que explique este elemento.

3.3.2. Casetas de la defensa antiaérea: También requieren limpieza y restauración, pues su estado no es muy bueno. Para poder acceder con facilidad es necesaria la construcción de unas escaleras en alguna de las pendientes. También aquí se instalaría un panel.

Epígrafes: Los Epígrafes (Fig. 8) necesitan ser consolidados y restaurados pues el soporto en piedra donde están escritos es frágil y se están deteriorando. Por lo tanto sería importante colocar una valla 


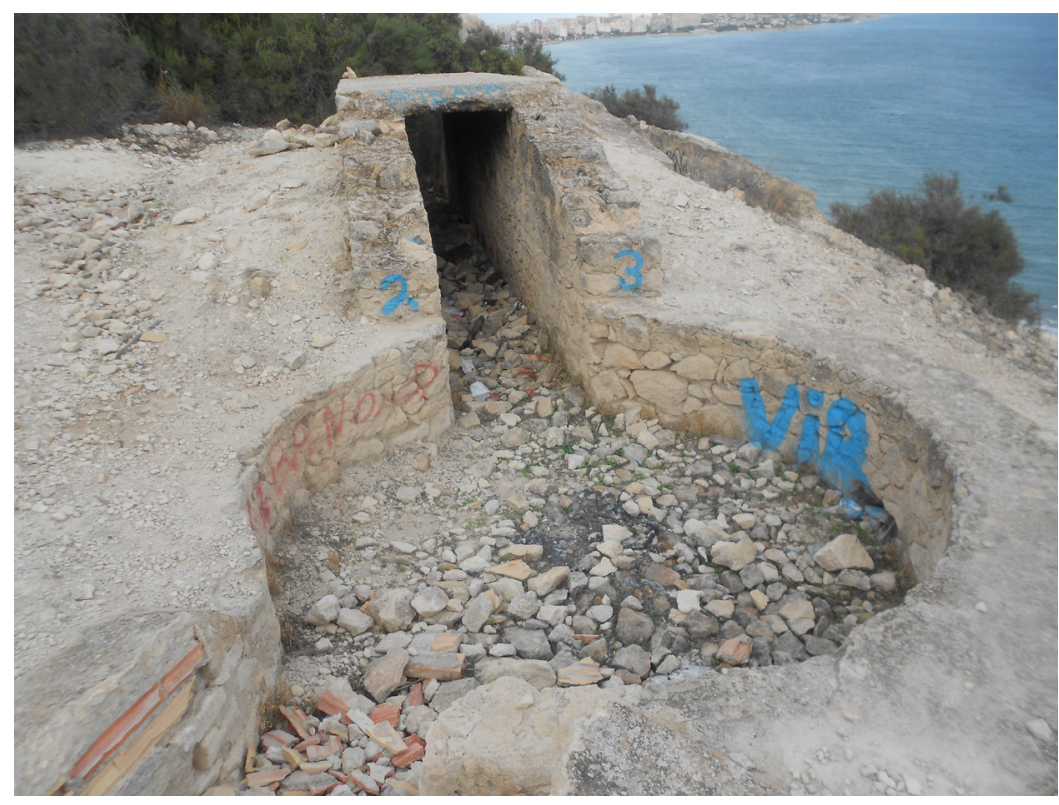

Fig. 7. Fotografía del nido de ametralladora. Fotografía de la autora.

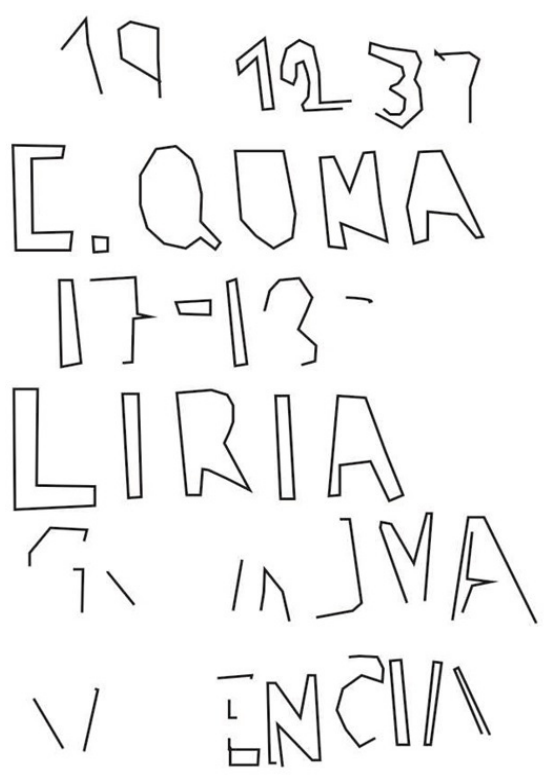

Fig. 8. Calco de epígrafes. Fuente: propia

que impida o al menos haga entender al visitante que no se deben tocar. Se instalará un panel con calcos para que se puedan observar mejor los epígrafes.

\subsection{El Polvorín}

El Polvorín no se construye en el periodo de la Guerra Civil, data del siglo XVIII, pero fue utilizado también como depósito de armas a lo largo del conflicto. Se encuentra en una de las laderas del Monte Benacantil, donde está el llamado Parque de la Ereta. Este lugar, durante la Guerra Civil, estuvo parte de la defensa antiaérea que defendía Alicante.

El Polvorín en la actualidad está en mal estado de conservación, no se puede visitar, por lo que sería necesario que fuera restaurado. En este lugar, se pondría un panel que explicara tanto la función del Polvorín como también la de la defensa antiaérea que allí se encuentra y otras defensas de la ciudad de las que no se conservan restos en la actualidad, como la del Castillo de San Fernando y otras que se encontraban por la ciudad.

\subsection{Mercado Central}

En el Mercado Central (Fig.9) se pueden observar algunos elementos que hacen referencia a la Guerra Civil, como son dos placas instaladas por el ayuntamiento y otra instalada por la Comisión Cívica de Alicante para la Recuperación de la Memoria Histórica. También está el reloj que marca la hora en la que se produjo el Bombardeo y la Sirena que no sonó a tiempo para avisar a la población. Junto a los elementos ya mencionados se pondría un panel un panel explicativo de los hechos acaecidos que se complementaria con las visitas guiadas.

\subsection{El Puerto}

La situación es similar a la del Mercado Central, solo hay un elemento que recuerda la tragedia allí sucedida y es la placa instalada en el 2014 por la Comisión Cívica de Alicante para la Recuperación de la Memoria Histórica. Para este lugar de propone la instalación del panel explicativo y visitas guiadas. 


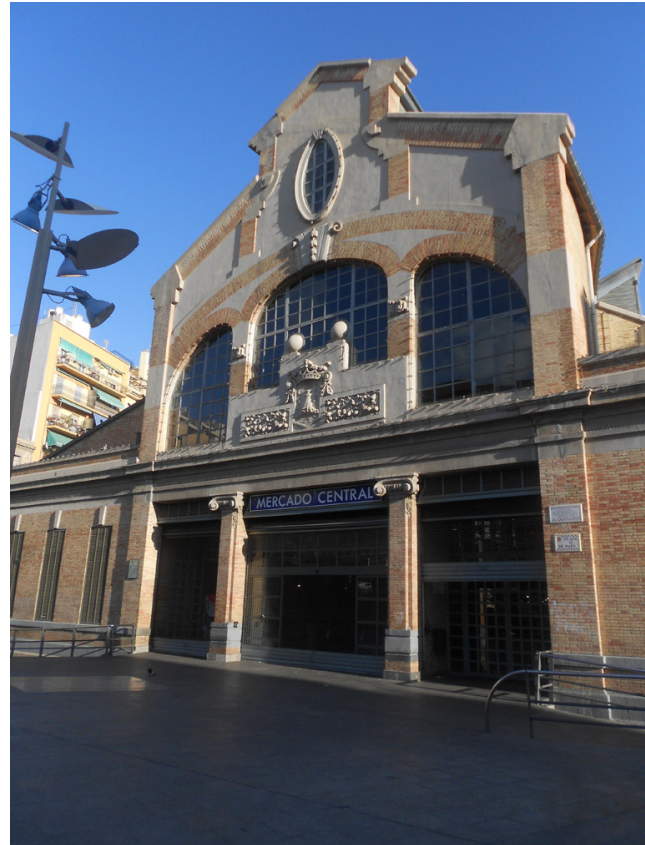

Fig. 9. Fotografía de la parte posterior del Mercado Central, donde se encuentran las placas que recuerdan el bombardeo ocurrido el 25 de mayo de 1938. Fotografía de la autora

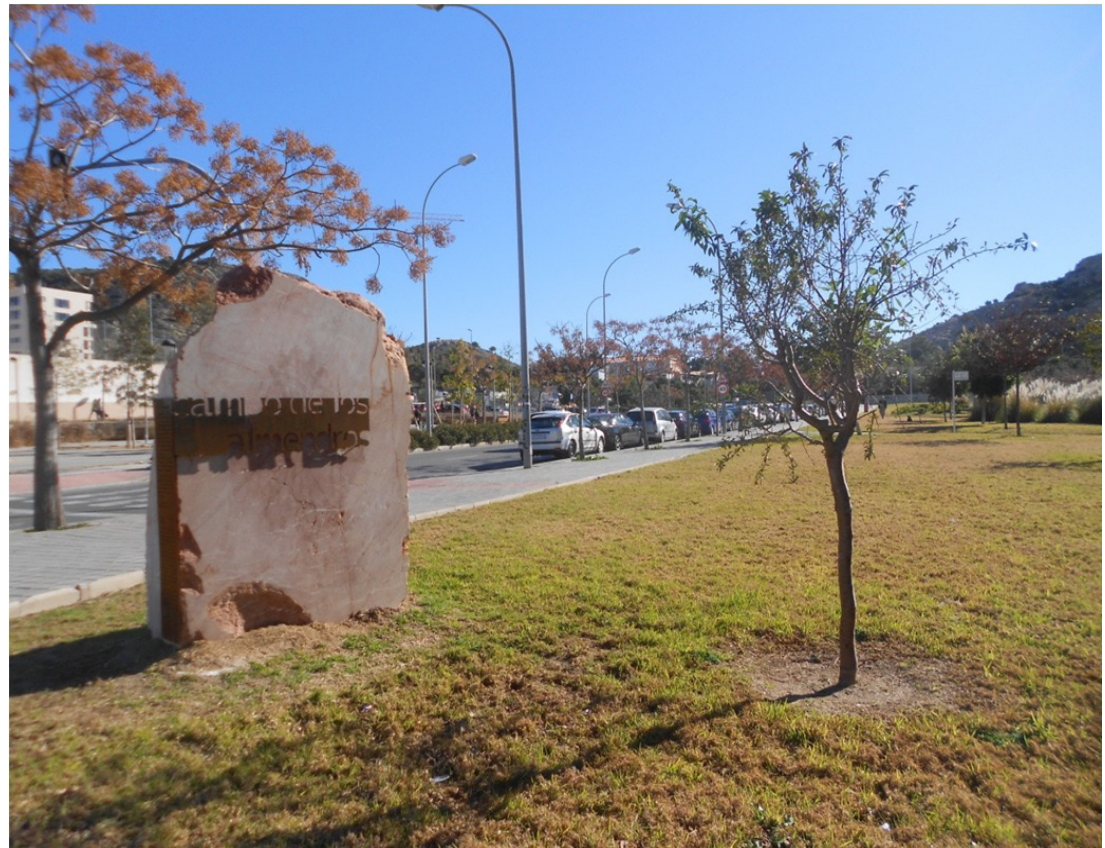

Fig. 10. Monumento conmemorativo. Fotografía de la autora.

\subsection{Campo de los Almendros}

Actualmente parte de lo que fue el Campo de los Almendros ha sido convertido en un parque con ese nombre; gracias a la Comisión Cívica de Alicante para la Recuperación de la Memoria Histórica se ha instalado allí un monolito (Fig.10) como homenaje a las personas que allí perdieron la vida. Para complementar esto se propone la instalación del panel perteneciente a la ruta.

\subsection{Graffitis Castillo}

Los graffitis realizados por los presos que estaban encarcelados en el Castillo de Santa Bárbara tras la Guerra Civil, se encuentran en un buen estado de conservación. No presentan ninguna tipo de medida de conservación, señalización o divulgación, y al encontrarse en el suelo pueden ser pisados por los visitantes del castillo ya que no constan de ningún tipo de protección .

Para su conservación y puesta en valor sería necesario en primer lugar colocar algún tipo de elemento que delimite la zona del suelo donde se encuentran los graffitis, para esto se podrían instalar unas placas de metal de entre unos 10 o $15 \mathrm{~cm}$ de altura que delimitaran la zona. De esta forma se llama la atención del visitante de que ahí hay algo y que al estar delimitado no se debería pasar ese perímetro, se podría instalar algún cartel que así lo advierta, así como el correspondiente panel explicativo.

En el otro extremo del Castillo de Santa Bárbara, en el macho del Castillo, se propone instalar un panel donde se explique los demás lugares de la ciudad a donde fueron llevados los prisioneros, pues desde esa altura se puede visualizar toda la ciudad. En el panel debe haber un dibujo de la vista de la ciudad desde ese ángulo donde aparezcan señalados los demás lugares utilizados como prisión (como la Plaza de Toros y el Castillo de San Fernando). 


\section{CONCLUSIONES}

Es fundamental abordar el periodo de la Guerra Civil desde una perspectiva arqueológica, que permita ampliar y complementar el discurso histórico respecto a dicho acontecimiento histórico. Así como acceder a elementos de conocimiento que no se podrían conocer de otra manera que no fuera mediante la arqueología (Alonso, 2008, p. 295).

Hay algunos elementos que caracterizan la puesta en valor de los restos de la Guerra Civil que no tienen los restos de otras épocas:

A) Elementos fácilmente distinguibles a simple vista, y comprensibles para el gran público.

B) Ha quedado, hasta ahora, fuera del campo de la investigación, ni la arqueología ni la historia contemporánea se ha ocupado de estos restos.

C) Ha quedado fuera de la protección patrimonial.

D) Por su cercanía temporal sigue teniendo una fuerte carga política. (Alonso, 2008, p.302).

Estos restos en muchas ocasiones necesitan ser estudiados más detalladamente y restaurados, pues muchos se encuentran en un estado lamentable de abandono y deterioro. Si no se hace algo por consolidarlos podría suponer la pérdida en unos años de este patrimonio, como sería el caso de las casetas de vigilancia de la Serra Grossa que cada vez están más deterioradas.

Con la creación del Centro de Interpretación de los "Refugios Antiaéreos" y la apertura al público de dos de estos refugios (el de la plaza Séneca y el de la plaza Balmis) se hace necesaria la creación de un itinerario que permita al visitante conocer otros lugares y restos materiales de la Guerra Civil, además de los refugios, para conocer lo sucedido así como otros aspectos de la guerra, como puede ser la defensa activa y la represión posterior a que la ciudad fuera tomada por las tropas fascistas. Hay que tener en cuenta la singularidad de este proyecto, pues, aunque en otras ciudades de nuestro país se han puesto en valor restos materiales de la Guerra Civil, en Alicante hay una gran variedad de este tipo de patrimonio (defensa activa y pasiva, sitios históricos, etc.) a una distancia relativamente corta, lo que permite crear una ruta fácil de realizar. Esto sumado al creciente interés por ese periodo de nuestra historia y su patrimonio hace que este proyecto pueda tener un gran atractivo para el turismo.

Es importante la creación de una mayor oferta cultural para el turista, además de la ya existente, como el MARQ y el yacimiento de Lucentum, que permita crear un turismo sostenible todo el año, que sea una alternativa y a la vez un complemento del turismo de sol y playa. También es importante no solo para el turismo, sino para la memoria de los propios alicantinos que tienen derecho a conocer su pasado, por lo que se debe fomentar el interés por este patrimonio y alentar su estudio. 


\section{BIBLIOGRAFÍA}

Aguilar Civera, I. (2006). De la Refinería La Británica a la factoría "La Cantera" de Alicante. Historia del Transporte, Obras Públicas y telecomunicaciones. Cuadernos del Museo del Transporte de la Comunidad Valenciana.

Alonso González, P. (2008). Reflexiones en torno a una Arqueología de la Guerra Civil: El caso de Laciana (León, España). Munibe (AntropologíaArkeologia), no 59. San Sebastián. pp. 291-312.

Archivo fotográfico de la Diputación Provincial de Alicante.

Bebía Llorca, P. (1997). Trabajo Graffiti. Castillo de Santa Bárbara. Trabajo inédito.

Besolí Martín, A; Peinado Cucarella, J. (2008). El estudio y puesta en valor de los refugios antiaéreos de la Guerra Civil Española: el caso del refugio-museo de Cartagena. Arqueomurcia. no 3. Consejería de Cultura y Turismo. Dirección General de Bellas Artes y Bienes Culturales. Servicio de Patrimonio Histórico.

Capdepón Carrascosa, L. (2006). Alicante Bombardeado (1936-1939). Instituto Alicantino de Cultura Juan Gil-Albert, Diputación Provincial de Alicante.

González Fraile, J. Navajas Corral, Óscar. (2011). Ley de Memoria Histórica: Estrategias para recuperar y comunicar el patrimonio de la Guerra Civil Española. Ebre, nำ 6. Asociación Espacios para la Memoria (AEP). Madrid. pp. 185-201.

González Ruibal, A. (2008). Arqueología de la Guerra Civil Española. Complutum, vol. 19, no 2. Despartamento de Prehistoria. Universidad Complutense de Madrid. Madrid. pp. 11-20.

Hernández Cardona, F. X. (2011). Conflictos contemporáneos, estrategias de musealización crítica. Museo y Territorio. no 4, pp. 79-86.

López Martínez, M. (2007). La experiencia republicana y la Guerra Civil en Alicante. Editorial Club Universitario, San Vicente, Alicante.
Lozano Olivares, F.; Lumbreras Voigt, M. (2015). Refugios antiaéreos de la Guerra Civil en Alicante: intervenciones arqueológicas en las plazas de Séneca y Dr. Balmis. Lucentum. No34, pp. 363-400.

Lozano Olivares, F. (2014). Seguimiento e intervención arqueológica. Proyecto de remodelación de la plaza dr. Balmis. Memoria. Concejalía de imagen urbana del Ayuntamiento de Alicante.

Martínez Mira, L. (2005). Alicante 1936.1939, Tiempos de Guerra. Alicante.

Moreno Sáez, F.; Martínez Leal, J.; Ors Montenegro, M.; Valero Escandell, J:R.; Moreno Seco, M. y Beneito Lloris, A. (2009). 1939 La Guerra se acaba en Alicante. Diario Información de Alicante. Alicante.

Muñoz Puelles, V. y Huguet, J. (2004). La conservación del patrimonio histórico militar de la Guerra Civil (1936-1939). Comissió de Promoció Cultural. Consell Valencià de Cultura.

Pérez Oca, M.A. (2006). 25 de mayo, la tragedia olvidada. Alicante.

Ramos Linaza, M. (2007). El turismo cultual, los museos y su planificación. Gijón.

Rosser Lamiñana, P. (2007). El Patrimonio Cultural de Alicante: Avance de un catálogo. El Patrimonio Inmueble. LQNT, Monográfico 3. Ayuntamiento de Alicante, Patronato Municipal de Cultura. Alicante.

VV.AA. (2003). Plan Especial de Protección Arqueológica del Término Municipal de Alicante (P.E.P.A.). Patronato Municipal de Alicante. Unidad de Conservación de Patrimonio HistóricoArtístico Municipal (COPHIAM). 


\section{RECURSOS WEB}

http://terrasit.gva.es/es/ver

http://sigpac.mapa.es/fega/visor/

http://www.catastro.meh.es/

http://fototeca.cnig.es/

https://www.google.es/maps http://restosguerracivilenalicante.blogspot. com.es/

https://comisioncivicalicante.wordpress.com/ 